\title{
Guía de Práctica Clínica: Nueva guía de cuidados preventivos de la Fuerza de Tareas de Estados Unidos (segunda entrega)
}

\begin{abstract}
Resumen
En 2001 la Fuerza de tareas Preventivas del EEUU (sigla en inglés: USPSTF) actualizó algunas recomendaciones de la segunda edición de su guía publicada en 1996' e incorporó algunas nuevas; lo que va constituyendo la tercera edición, a la que también se puede acceder gratuita y completamente en el sitio: http://www.ahrq.gov/clinic/uspstfix.htm

Ofrecemos en esta segunda entrega un resumen de las recomendaciones preventivas publicadas desde 2003 y su justificación clínica complementando la primera entrega ${ }^{2}$. En dicha entrega de EVIDENCIA hemos resumido las recomendaciones sobre consejería, indicación de suplementos y rastreo de problemas relacionados con estilos de vida. En esta segunda entrega nos explayamos sobre las recomendaciones de rastreo del resto de las condiciones clínicas. No hemos incluido las inmunizaciones y la profilaxis post exposición de enfermedades infecciosas pues ambas están regidas por las Normas Nacionales de Vacunación 2004 (http://www.direpi.vigia.org.ar/pdf/Normas_nacionales_03-2004.pdf).
\end{abstract}

Adaptado por Ciapponi A de la. Nueva guía de cuidados preventivos de la Fuerza de Tareas de Estados Unidos (segunda entrega). Evid. actual. práct. ambul; 9(1): 26-30, Ene-Feb 2006.

En la tabla a continuación se observa un resumen de las recomendaciones para cada problema de salud, complementando la entrega previa. En el texto que le sigue se justifican dichas recomenda- ciones. Las presentes entregas complementan a su vez una actualización anterior publicada en Evidencia que incluyó el material generado por la USPSTF entre 2000 y $2003^{3}$.

Tabla: Tabla. Recomendaciones preventivas seleccionadas de la Fuerza de Tareas de Estados Unidos (USPSTF).

\begin{tabular}{|c|c|c|c|c|}
\hline Problema & \multicolumn{2}{|l|}{ Práctica Preventiva } & Recom. & Publ. \\
\hline \multirow{3}{*}{$\begin{array}{l}\text { Aneurisma de Aorta Abdominal } \\
\text { (AAA) }\end{array}$} & \multicolumn{2}{|c|}{ mediante una ecografía en hombres de 65 a 75 años que hayan fumado } & $\mathbf{B}^{1}$ & \multirow{3}{*}{$2 / 2005$} \\
\hline & \multirow{2}{*}{\multicolumn{2}{|c|}{$\begin{array}{l}\text { en hombres de } 65-75 \text { años que nunca hayan fumado } \\
\text { en mujeres }\end{array}$}} & $\mathbf{C}^{2}$ & \\
\hline & & & $\mathbf{D}^{3}$ & \\
\hline Aerteriopatía periférica & \multicolumn{2}{|c|}{ en población general adulta mediante el índice tobillo-brazo (doppler) } & $\mathrm{D}^{4}$ & $8 / 2005$ \\
\hline Demencia & \multicolumn{2}{|l|}{ en población general } & $I^{5}$ & $6 / 2003$ \\
\hline \multirow[t]{2}{*}{ Hipertensión Arterial } & \multicolumn{2}{|l|}{ en adultos $\geq 18$ años } & $\mathbf{A}^{6}$ & \multirow[b]{2}{*}{$7 / 2003$} \\
\hline & \multicolumn{2}{|l|}{ en niños y adolescentes } & $\mathbf{P}^{7}$ & \\
\hline \multirow{3}{*}{ Gonorrea } & \multicolumn{2}{|l|}{ en mujeres asintomáticas de alto riesgo } & $\mathrm{B}^{8}$ & \multirow{3}{*}{$5 / 2005$} \\
\hline & \multicolumn{2}{|l|}{ a toda embarazada o a hombres asintomáticos de alto riesgo } & $I^{9}$ & \\
\hline & \multicolumn{2}{|l|}{ en población general adulta } & $\mathbf{D}^{10}$ & \\
\hline \multirow[t]{2}{*}{ Hepatitis B } & \multicolumn{2}{|l|}{ en embarazadas en su 1er control prenatal } & $\overline{A^{11}}$ & \multirow[t]{2}{*}{$2 / 2004$} \\
\hline & \multicolumn{2}{|l|}{ en población general } & $\overline{\mathbf{D}^{12}}$ & \\
\hline \multirow[t]{2}{*}{ Hepatitis C } & \multicolumn{2}{|l|}{ en población general } & $\overline{\mathbf{D}^{13}}$ & \multirow[b]{2}{*}{$3 / 2004$} \\
\hline & \multicolumn{2}{|l|}{ en adultos de riesgo } & $\mathbf{I}^{14}$ & \\
\hline Cáncer vesical & \multicolumn{2}{|l|}{ en adultos } & $\overline{\mathbf{D}^{15}}$ & $6 / 2004$ \\
\hline Cáncer de pulmón & $\begin{array}{l}\text { mediante tomografía computada de baja dosis, } \mathrm{Rx} \text { de } \\
\text { esputo o combinándolos }\end{array}$ & tórax, citología de & $I^{16}$ & $5 / 2004$ \\
\hline Cáncer de ovario & mediante CA-125 sérico o ecografía transvaginal & & $\overline{D^{17}}$ & $5 / 2004$ \\
\hline & mediante consejo genético y búsqueda de mutación & alto & $\mathbf{B}^{18}$ & \\
\hline & del gen BRCA según historia familiar de riesgo & promedio & $\mathbf{D}^{19}$ & $9 / 2005$ \\
\hline Cáncer de mama & mediante consejo genético y búsqueda de mutación del & alto & $\mathbf{B}^{18}$ & \\
\hline & gen BRCA según historia familiar de riesgo & promedio & $\mathbf{D}^{19}$ & $9 / 2005$ \\
\hline Cáncer oral & en adultos & & $1^{20}$ & $2 / 2004$ \\
\hline Cáncer de páncreas & $\begin{array}{l}\text { en adultos asintomáticos mediante palpación abdomina } \\
\text { cadores serológicos }\end{array}$ & , ecografía o mar- & $\overline{D^{21}}$ & $2 / 2004$ \\
\hline Cáncer de testículo & en adolescentes y adultos asintomáticos & & $\overline{D^{22}}$ & $2 / 2004$ \\
\hline Sífilis & en embarazadas y personas de riesgo de sífilis & & $\mathrm{A}^{23}$ & $7 / 2004$ \\
\hline & en personas asintomáticas de bajo riesgo de sífilis & & $\mathbf{D}^{24}$ & \\
\hline Bacteriuria asintomática & en embarazadas en semana 12 a 16 mediante urocultivo & & $A^{25}$ & \\
\hline & en hombres y mujeres no embarazadas & & $\mathbf{D}^{26}$ & $2 / 2004$ \\
\hline Enfermedad coronaria & en adultos de bajo riesgo de eventos coronarios & & $\mathbf{D}^{27}$ & \\
\hline & en adultos de riesgo de eventos coronarios & & $\mathbf{I}^{28}$ & $2 / 2004$ \\
\hline Herpes genital & en embarazadas asintomáticas mediante serología & & $\mathrm{I}^{29}$ & \\
\hline & en adolescentes asintomáticas y adultos mediante serolc & gía & $\mathbf{D}^{30}$ & $3 / 2005$ \\
\hline Glaucoma & en adultos mediante la medición de la presión intraocula & $(\mathrm{PIO})$ & $\mathbf{I}^{31}$ & $3 / 2005$ \\
\hline Sobrepeso & en niños y adolescentes & & $\overline{T^{32}}$ & $7 / 2005$ \\
\hline Enfermedad tiroidea & en adultos & & $\mathbf{1}^{33}$ & $1 / 2004$ \\
\hline Incompatibilidad Rh & $\begin{array}{l}\text { en embarazadas en su 1er control prenatal mediante } \\
\text { Anticuerpos Anti-Rh }\end{array}$ & tipificación Rh y & $\begin{array}{l}A^{34} \\
B^{35}\end{array}$ & $2 / 2004$ \\
\hline & repetir rastreo a las $24-28$ semanas excepto con padre $\mathrm{F}$ & & & \\
\hline Visual & niños menores de 5 años & & $\mathrm{B}^{36}$ & $3 / 2004$ \\
\hline Caries & $\begin{array}{l}\text { en niños preescolares mayores de } 6 \text { meses de zonas cc } \\
\text { ración del agua }\end{array}$ & insuficiente fluo- & $\mathbf{B}^{37}$ & $4 / 2004$ \\
\hline & en niños preescolares mediante valoración de riesgo & & $\mathbf{I}^{38}$ & \\
\hline Escoliosis idiopática & en adolescentes & & $\overline{D^{39}}$ & $6 / 2004$ \\
\hline
\end{tabular}


1- AAA en hombres de 65-75 años que hayan fumado mediante 1 ecografía (B)

Hay buena evidencia que el rastreo de AAA y la reparación quirúrgica de AAA grandes ( $\geq 5 \mathrm{~cm}$.) en hombres de 65 a 75 años que hayan fumado alguna vez (fumadores actuales y ex-fumadores) disminuye la mortalidad específica por AAA. Hay buena evidencia de que la ecografía abdominal (con adecuada garantía de calidad), es una prueba segura para el rastreo de AAA. Hay también buena evidencia de daños importantes del rastreo y tratamiento temprano, incluyendo un número aumentado de cirugías y la consecuente morbimortalidad asociada y daños psicológicos a corto plazo. Basado en la magnitud moderada del beneficio neto, se concluyó que los beneficios de rastrear AAA en los hombres de 65 a 75 años que hayan fumado alguna vez superan a los daños.

2- AAA en hombres de 65-75 años que nunca hayan fumado (C) Hay buena evidencia que el rastreo de AAA en hombres de 65 a 75 años que nunca fumaron disminuye la mortalidad específica por AAA. Sin embargo, hay una menor prevalencia de AAA en este grupo por lo que el beneficio es pequeño. Hay también buena evidencia de daños importantes del rastreo y tratamiento temprano, incluyendo un número aumentado de cirugías y la consecuente morbimortalidad asociada y daños psicológicos a corto plazo. Basado en la escasa magnitud del beneficio neto, se concluyó que el balance entre beneficios y daños de rastrear AAA en los hombres de 65 a 75 años que nunca hayan fumado es muy estrecho como para recomendarlo a esta población.

\section{3- AAA en mujeres (D)}

Dada la baja prevalencia de AAA en mujeres, el número de muertes por AAA prevenibles por rastreo es muy bajo. Hay buena evidencia de daños importantes del rastreo y tratamiento temprano, incluyendo un número aumentado de cirugías y la consecuente morbimortalidad asociada y daños psicológicos a corto plazo. Se concluyó que los daños de rastrear AAA en mujeres superan a los beneficios.

4- Arteriopatía periférica en población general adulta mediante el índice tobillo-brazo (doppler) (D)

Hay aceptable evidencia de que el rastreo mediante el índice tobillo-brazo puede detectar arteriopatía periférica asintomática pero dada su baja prevalencia en la población general adulta y la escasa evidencia de tratamientos adicionales al control de los factores de riesgo cardiovasculares, dicho rastreo tendría beneficios pobres o nulos. Hay aceptable evidencia de que el rastreo mediante el índice tobillo-brazo podría generar daños menores, incluyendo los falsos positivos y cascadas diagnósticas innecesarias.

Se concluyó que los daños de rastrear AAA en mujeres superan a los beneficios.

\section{5- Demencia (I)}

Se encontró buena evidencia de que algunas pruebas tienen buena sensibilidad ${ }^{*}$ pero solo moderada especificidad ${ }^{*}$ para detectar deterioro cognitivo y demencia. Hay de moderada a buena evidencia de que varias drogas pueden mejorar la función cognitiva (demora de 2 a 7 meses en la progresión de la enfermedad de Alzheimer), pero la evidencia de efectos benéficos en actividades de la vida diaria, pequeños en el mejor de los casos, es contrapuesta.

Se encontró insuficiente evidencia como para determinar si los beneficios encontrados en los ensayos terapéuticos pueden ser extrapolables a pacientes detectados por rastreo en ámbitos de atención primaria. Se desconoce la precisión diagnóstica, factibilidad del rastreo y del tratamiento en la práctica clínica, así como los daños potenciales del rastreo (Ej: efecto de etiquetamiento). No se puede determinar el balance entre beneficios y daños del rastreo rutinario para demencia.
6- Hipertensión Arterial (HTA) en adultos $\geq 18$ años (A) Hay buena evidencia de que la medición de la tensión arterial puede identificar adultos con riesgo de enfermedad cardiovascular aumentado por HTA y buena evidencia de que el tratamiento reduce su incidencia ocasionando pocos daños mayores. Los beneficios de rastrear y tratar HTA en adultos superan sustancialmente los daños.

\section{7- HTA en niños y adolescentes (I)}

Hay pobre evidencia de que la medición de la tensión arterial puede identificar con exactitud a niños y adolescentes con riesgo de enfermedad cardiovascular aumentado, y pobre evidencia de que el tratamiento reduce su incidencia. No puede determinarse el balance entre beneficios y daños de rastrear HTA en niños $y$ adolescentes.

8- Gonorrea en mujeres asintomáticas de alto riesgo (B)

Se recomienda el rastreo de Neisseria gonorrhoeae en mujeres asintomáticas de alto riesgo de infección (embarazadas o no embarazadas) preferentemente mediante cultivo endocervical.

\section{9- Gonorrea a embarazada de bajo riesgo o a hombres asin-} tomáticos de alto riesgo (I)*

Hay insuficiente evidencia como para recomendar el rastreo a toda embarazada o a hombres asintomáticos de alto riesgo. Podrían ser rastreados los hombres jóvenes de alto riesgo sexualmente activos para tratarlos y así prevenir la transmisión a sus parejas. El rastreo a hombres con tiras reactivas de estearasa leucocitaria en orina es conveniente y barato pero requiere la confirmación de resultados positivos.

\section{0- Gonorrea en población general adulta (D)}

No se recomienda el rastreo en población general adulta.

11- Hepatitis $B$ en embarazadas en su 1er control prenatal (A) Hay buena evidencia de que el rastreo prenatal de Hepatitis B mediante $\mathrm{HBsAg}$ reduce su transmisión prenatal y el desarrollo de Hepatitis B crónica. La vacunación de neonatos contra la Hepatitis B y la profilaxis postexposición con gamaglobulina hiperinmune específica a neonatos de madres infectadas reduce el riesgo de adquirir la Hepatitis B.

\section{2- Hepatitis B en población general (D)}

No hay evidencia de que el rastreo de Hepatitis B en la población general mejore los resultados a largo plazo como la cirrosis, carcinoma hepatocelular o la mortalidad. La prevalencia de Hepatitis B es baja, la mayoría de los infectados no progresan a la cronicidad, cirrosis o hepatopatía por Hepatitis B. Los daños potenciales del rastreo incluyen al etiquetamiento pero la evidencia en cuanto a su magnitud es limitada. Los daños potenciales del rastreo de Hepatitis $B$ en la población general probablemente excedan los beneficios.

\section{3- Hepatitis $C$ en población general (D)}

Hay buena evidencia de que el rastreo puede detectar Hepatitis $C$ en la población general. La prevalencia de Hepatitis C es baja, la mayoría de los infectados no desarrollan cirrosis o resultados adversos mayores. No hay evidencia de que el rastreo de Hepatitis $\mathrm{C}$ mejore los resultados a largo plazo como la cirrosis, carcinoma hepatocelular o la mortalidad. Aunque hay buena evidencia de que el tratamiento antiviral mejora resultados intermedios como viremia, hay poca evidencia de resultados a largo plazo. El tratamiento es largo, costoso y muchos lo abandonan por efectos adversos. Los daños potenciales del rastreo incluyen biopsias innecesarias y etiquetamiento. Es por ello que los daños potenciales del rastreo de 
Hepatitis $C$ en la población general sin riesgo probablemente excedan los beneficios.

\section{4- Hepatitis $C$ en adultos de riesgo (I)}

No se encontró evidencia que el rastreo de Hepatitis $\mathrm{C}$ en adultos de riesgo (adictos intravenosos; transfusión antes de 1990; diálisis; ser hijo de una madre con Hepatitis $\mathrm{C}$; conducta sexual de alto riesgo y el uso de drogas ilegales, como cocaína o marihuana) mejore los resultados a largo plazo, aunque el rédito sería mucho mayor que en una población de riesgo habitual. Hay buena evidencia de que el tratamiento antiviral mejora resultados intermedios como viremia, pero no hay evidencia de que los nuevos tratamientos (como el interferón pegilado y ribavirina) mejoren los resultados a largo plazo. Hay limitada evidencia sobre los beneficios a largo plazo de otros tratamientos, y su extrapolación a la población general es desconocida. Hay limitada evidencia de que 10 a $20 \%$ de los pacientes con Hepatitis C crónica desarrollan cirrosis 20 a 30 años después de la infección. Hay también evidencia limitada que los tratamientos disponibles son eficaces previniendo la cirrosis en los pacientes con Hepatitis C asintomática. Los daños potenciales de rastrear y tratar incluyen el etiquetamiento, efectos adversos por el tratamiento y las biopsias innecesarias, aunque hay limitada evidencia como para determinar la magnitud de estos daños. No puede determinarse el equilibrio entre beneficios y daños del rastreo de Hepatitis $C$ en adultos de riesgo de Hepatitis $C$.

\section{5- Cáncer vesical en adultos (D)}

Hay aceptable evidencia de que el rastreo con las pruebas disponibles puede descubrir el cáncer vesical en individuos asintomáticos. El beneficio potencial del rastreo sería pequeño, en el mejor de los casos, por las siguientes razones: hay aceptable evidencia que muchos de los cánceres descubiertos por rastreo tienen una baja tendencia a progresar a enfermedad invasiva; hay una relativamente baja prevalencia global de cáncer vesical generador de consecuencias clínicas importantes y hay evidencia limitada de que el tratamiento temprano del cáncer vesical descubierto por rastreo mejore los resultados de salud a largo plazo. Los daños potenciales de rastrear son por lo menos pequeños: las pruebas tienen un bajo valor predictivo positivo* y da muchos resultados falsos positivos, que conducen a procedimientos invasivos innecesarios. Los daños potenciales de rastrear el cáncer vesical superan cualquier beneficio potencial.

16- Cáncer de pulmón mediante tomografía computada de baja dosis (TCBD), Rx de tórax, citología de esputo o combinándolos (I)

Se encontró aceptable evidencia de que el rastreo con TCBD, Rx de tórax o citología de esputo puede descubrir el cáncer pulmonar en una fase más temprana de lo que se descubriría en una población no rastreada; sin embargo, la evidencia es pobre de que cualquier estrategia disminuya la mortalidad. Debido a la naturaleza invasiva de diagnóstico y la posibilidad de un alto número de pruebas falso-positivas en ciertas poblaciones, hay potencial para producir daños significativos con el rastreo. No puede determinarse el equilibrio entre los beneficios y daños de rastrear el cáncer pulmonar.

\section{7- Cáncer de ovario mediante CA-125 sérico o ecografía transvaginal (D)}

Se encontró aceptable evidencia de que el rastreo con CA-125 sérico o ecografía transvaginal puede descubrir el cáncer ovárico en una fase más temprana que sin rastreo; sin embargo, también hay aceptable evidencia de que la detección temprana tendría un efecto probablemente pequeño, en el mejor de los casos, sobre la mortalidad del cáncer ovárico. Debido a la baja prevalencia de cáncer ovárico y la naturaleza invasiva del diagnóstico después de una prueba de rastreo positiva, hay aceptable evidencia de que el rastreo podría implicar a daños importantes. Los daños potenciales superan a los beneficios potenciales.

18- Cáncer de ovario mediante consejo genético y búsqueda de mutación del gen BRCA con historia familiar de alto riesgo (B)

Se encontró aceptable evidencia de que las mujeres con historia familiar de riesgo tienen un mayor riesgo de desarrollar cáncer de mama u ovario asociados a la mutación genética BRCA1 o BRCA2. Se considera historia familiar de riesgo: 2 familiares de 1er grado con cáncer mamario (1 de éstos con diagnóstico $\leq 50$ años); $\geq 3$ familiares de 1er o 2 do grado con cáncer mamario independientemente de la edad al diagnóstico; familiares de 1er o 2do grado con cáncer mamario y ovárico; un familiar de 1er grado con cáncer mamario bilateral; $\geq 2$ familiares de 1 er o 2 do grado con cáncer ovárico independientemente de la edad al diagnóstico; cáncer mamario en familiar varón.

Estas mujeres se beneficiarían de un consejo genético por personal entrenado que permita una decisión informada sobre el test y los tratamientos profilácticos. Hay insuficiente evidencia de que la quimioprofilaxis o rastreos intensivos mejoren los resultados en salud de las mujeres portadoras de las mutaciones BCRA, pero hay bastante evidencia de que la cirugía profiláctica reduce la incidencia de cáncer ovárico/mamario. Hay insuficiente evidencia sobre las consecuencias éticas legales y sociales de derivar a consejo genético seguido del test, pero los riesgos de la cirugía profiláctica son conocidos. Los beneficios de derivar a mujeres con historia familiar de riesgo para un consejo genético provisto por personal capacitado superan los daños potenciales.

19- Cáncer de ovario mediante consejo genético y búsqueda de mutación del gen BRCA según historia familiar de riesgo promedio (D)

Se encontró aceptable evidencia de que las mujeres sin historia familiar de riesgo tienen un bajo riesgo de desarrollar cáncer de mama u ovario asociados a la mutación genética BRCA1 o BRCA2 por lo que los beneficios del rastreo serían pequeños o nulos. Considerando los riesgos conocidos ya mencionados se concluyó que los daños potenciales superan a los beneficios potenciales.

\section{0- Cáncer oral en adultos (I)}

No se encontró ninguna nueva evidencia de buena calidad que el rastreo de cáncer oral mejore los resultados en adultos de riesgo del contraerlo (Ej: fumadores de más de 50 años) o en la población general adulta de riesgo promedio. Es improbable que alguna vez se realicen ensayos controlados de rastreo de cáncer oral en la población general debido a la muy baja incidencia del mismo. Tampoco hay nuevas evidencias de los daños del rastreo. No puede determinarse el equilibrio entre los beneficios y daños de rastrear el cáncer oral.

21- Cáncer de páncreas en adultos asintomáticos mediante palpación abdominal, ecografía o marcadores serológicos (D) No se encontró ninguna evidencia de que rastrear el cáncer pancreático sea eficaz reduciendo la mortalidad. Hay un potencial daño significativo debido a la prevalencia muy baja de cáncer pancreático, exactitud limitada de pruebas de rastreo disponibles, la naturaleza invasiva del diagnóstico después de una prueba de rastreo positiva, y los pobres resultados del tratamiento. Los daños de rastrear el cáncer pancreático exceden cualquier beneficio potencial.

\section{2- Cáncer de testículo en adolescentes y adultos asintomáti-} $\cos (\mathrm{D})$

No se encontró ninguna nueva evidencia de que el rastreo mediante el examen clínico o el autoexamen testicular sea eficaz reduciendo la mortalidad por cáncer testicular. Incluso en la ausencia de rastreo, el tratamiento actual proporciona resultados de salud muy favorables. Dada la baja prevalencia del cáncer testicu- 
lar, la exactitud limitada de las pruebas de rastreo y la falta de evidencias de beneficios incrementales de rastrear, se concluyó que los daños de rastrear exceden cualquier beneficio potencial.

23- Sífilis en embarazadas y personas de riesgo de sífilis (A) No se encontró ninguna nueva evidencia directa de que el rastreo de infección sifilítica mejore los resultados de salud en las personas de alto riesgo (hombres que tienen sexo inseguro con hombres, trabajadores del sexo, personas que intercambian sexo por drogas, adultos en correccionales o cárceles y posiblemente personas con otra enfermedad de transmisión sexual). Sin embargo hay adecuada evidencia de que las pruebas de rastreo no treponémicas (VDRL o RPR) seguidas de la confirmación con las treponémicas (FTA-ABS o TP-PA), pueden detectar la infección sifilítica con precisión y que los antibióticos pueden curarla. Se desconoce el intervalo de rastreo óptimo en personas de alto riesgo. Rastrear puede producir daños potenciales (como la evaluación clínica de resultados falso-positivos, ansiedad innecesaria al paciente y daños por el uso antibiótico). Los beneficios de rastrear a personas de alto riesgo de infección sifilítica superan a los daños potenciales.

Hay evidencia observacional de que el rastreo universal en embarazadas en su primer control prenatal reduce la proporción de bebés con manifestaciones clínicas de sífilis y con serologías positivas. Los beneficios de rastrear infección sifilítica a mujeres embarazadas superan sustancialmente a los daños potenciales.

\section{4- Sífilis en personas asintomáticas de bajo riesgo de sífilis} (D)

Dado la baja incidencia de infección sifilítica en la población general y el consecuente bajo rédito del rastreo se concluye que el potencial daño de rastrear (es decir, el costo de oportunidad, las pruebas falso-positivas, y etiquetamiento) supera a los beneficios.

\section{5- Bacteriuria asintomática en embarazadas en semana 12 a} 16 mediante urocultivo (A)

Hay buena evidencia de que rastrear bacteriuria asintomática en embarazadas en semana 12 a 16 mediante urocultivo reduce significativamente las infecciones urinarias sintomáticas, el bajo peso al nacer y los recién nacidos pretérmino. Los beneficios de rastrear y tratar superan sustancialmente cualquier daño potencial.

\section{6- Bacteriuria asintomática en hombres y mujeres no embarazadas (D)}

Hay aceptable evidencia de que el rastreo de bacteriuria asintomática a hombres y mujeres no embarazadas es ineficaz mejorando resultados clínicos. En la ausencia de evidencia de beneficio, los daños potenciales asociados con el sobreuso de antibióticos son especialmente significativos.

\section{7- Enfermedad coronaria en adultos de bajo riesgo de even-} tos coronarios (D)

Hay por lo menos aceptable evidencia de que el ECG o la prueba ergométrica (PEG) pueden descubrir a algunos adultos asintomáticos con riesgo aumentado de eventos coronarios independientemente de los factores de riesgo coronarios (FRC). La PEG puede detectar obstrucción coronaria severa en un pequeño número de adultos asintomáticos. Hay limitada evidencia similar para la TC por haz de electrones (electron-beam computerized tomography [EBCT]). En ausencia de evidencia de que la detección por ECG, PEG, O EBCT en adultos de bajo riesgo de eventos coronarios finalmente mejore los resultados de salud y que probablemente las pruebas falso-positivas causen daño debido a procedimientos invasivos innecesarios, sobretratamientos y etiquetamiento, se concluyó que los daños potenciales del rastreo de rutina de enfermedad coronaria en esta población exceden los beneficios potenciales. Consideraciones Clínicas: la valoración de riesgo debe investigar la presencia y severidad de los siguientes factores de riesgo: edad, sexo, diabetes, colesterol total (3200mg \%), HDLc (<50 mg\%), presión arterial (3130/85), antecedentes familiares (en familiares adultos jóvenes), obesidad (IMC $>30 \mathrm{Kg} / \mathrm{m} 2$ ), sedentarismo (menos de 30 minutos 3 veces por semana de actividad aeróbica) y tabaquismo (al menos 1 cigarrillo en el último mes). Hay herramientas que proporcionan una valoración más exacta del riesgo cardiovascular (un ejemplo en español es la calculadora del Foro APS, http://200.47.22.81/riesgo/). Para las personas en ciertas ocupaciones, como pilotos y los operadores de equipos peligrosos (para quien un síncope o la muerte súbita pueden poner en peligro la seguridad de otros), podría decidirse el rastreo no ya basado en el paciente individual. Aunque algunos programas de ejercicio rastrean inicialmente a participantes asintomáticos con PEG, no hay suficiente evidencia como para determinar el equilibrio de beneficios y daños de esta práctica.

\section{8- Enfermedad coronaria en adultos de alto riesgo de eventos} coronarios (I)

Hay inadecuada evidencia como para determinar hasta que punto la detección adicional a la valoración de FRC provista por el ECG, PEG o EBCT mejoraría los resultados de salud relacionados a enfermedad coronaria en adultos de alto riesgo de eventos coronarios. Aunque hay limitada evidencia como para determinar la magnitud de daños por rastrear a esta población, es probable que existan daños por las pruebas falso-positivas. No se puede determinar el equilibrio entre los beneficios y daños de rastrear a esta población para enfermedad coronaria.

\section{9- Herpes genital en embarazadas asintomáticas mediante} serología (D)

Hay aceptable evidencia de que el rastreo en embarazadas asintomáticas mediante serología no reduce la transmisión de Virus Hespes Simplex (VHS) a neonatos. Las mujeres que desarrollan infección primaria por VHS tienen mayor riesgo de transmisión de la infección a sus hijos. Dado que estas mujeres son inicialmente seronegativas, el rastreo serológico de VHS mediante ELISA o western blot no detectan con precisión a las mujeres de mayor riesgo. No hay evidencia de que tratar a las mujeres seronegativas reduzca el riesgo de infección neonatal. Hay limitada evidencia de que la terapia antiviral en mujeres con VHS recu-rrente, o que la cesárea en mujeres con lesiones activas por VHS al momento del parto, reduzca el herpes neonatal. También es limitada la evidencia de la seguridad de la terapia antiviral en embarazadas y neonatos. Los daños potenciales del rastreo, aunque no bien estudiados, incluyen resultados falsos positivos, etiquetamiento, ansiedad, así como falsos negativos y falso reaseguro. Se concluyó que no hay beneficios asociados al rastreo por los que daños potenciales superan los beneficios.

30- Herpes genital en adolescentes asintomáticas y adultos mediante serología (D)

No se hallo evidencia de que el rastreo en adolescentes asintomáticas y adultos mediante serología mejore los resultados en salud, los síntomas o reduzca la transmisión de la enfermedad. Hay buena evidencia de que la serología puede identificar con exactitud de las personas expuestas al VHS. Hay buena evidencia de que la terapia antiviral mejora resultados en salud en personas sintomáticas (Ej: con múltiples recurrencias) pero no hay evidencias para personas asintomáticas. Los daños potenciales del rastreo incluyen resultados falsos positivos, etiquetamiento, ansiedad, aunque la evidencia es limitada de los daños potenciales del rastreo o del tratamiento. Se concluyó que los beneficios del rastreo son, en el mejor de los casos, mínimos y que los daños potenciales superan los beneficios.

31- Glaucoma en adultos mediante la medición de la presión intraocular (PIO) (I)

Se concluyó que la evidencia es insuficiente como para hacer una 
recomendación a favor o en contra de rastrear el glaucoma.

Se encontró buena evidencia de que el rastreo puede detectar presión intraocular (PIO) elevada y glaucoma primario temprano de ángulo abierto (GPTAA) en adultos. También se encontró buena evidencia de que el tratamiento en adultos con PIO elevada detectada por rastreo reduce el número de personas que desarrollan pequeños defectos del campo visual y que el tratamiento temprano del GPTAA asintomático reduce la progresión de los defectos visuales. Sin embargo la evidencia es insuficiente como para determinar en que grado la detección temprana de PIO elevada y GPAA podría reducir el deterioro de la función visual y la calidad de vida. Por otro lado, hay buena evidencia de que el tratamiento de la PIO elevada y GPTAA produce daños como irritación ocular y riesgo de cataratas.

Dada la incertidumbre de la magnitud de beneficio del tratamiento temprano y los conocidos daños del rastreo y tratamiento temprano, no se puede determinar el equilibrio entre los beneficios y daños de rastrear glaucoma.

\section{2- Sobrepeso en niños y adolescentes (I)}

Los niños y adolescentes de 6-19 años con sobrepeso tienen mayor riesgo de sobrepeso futuro, dislipemia, hipertensión, esteatohepatitis, lesión de epífisis femoral, apnea del sueño y problemas psicosociales. Hay aceptable evidencia de que el Índice de Masa Corporal (IMC) para identificar el sobrepeso es útil y se asocia a un mayor riesgo de obesidad en la adultez. Hay insuficiente evidencia de la efectividad o daños del consejo conductual u otras intervenciones preventivas en niños y adolescentes con sobrepeso en ámbitos de atención primaria. Se concluyó que la evidencia es insuficiente como para hacer una recomendación a favor o en contra de rastrear niños y adolescentes con sobrepeso.

\section{3- Enfermedad tiroidea en adultos (I)}

Hay aceptable evidencia de que el dopaje de TSH puede detectar enfermedad tiroidea subclínica, pero pobre evidencia de que el tratamiento produzca una mejoría clínicamente significativa de resultados de las patologías tiroideas detectadas por rastreo. Aunque el rédito del rastreo es mayor en ciertos grupos de riesgo (Ej: mujeres posparto, pacientes con Síndrome de Down y ancianos), se halló pobre evidencia de que rastrear estos grupos produzca beneficios clínicos importantes. Existen daños potenciales debido a resultados falso-positivos pero la magnitud del mismo se desconoce. Hay buena evidencia de que el sobretratamiento con levotiroxina es común, pero se desconocen sus efectos nocivos a largo plazo. No puede determinarse el equilibrio entre beneficios y daños de rastrear enfermedad tiroidea subclínica en adultos.

34- Incompatibilidad Rh, en embarazadas en su 1er control prenatal (A)

Hay buena evidencia de que tipificar el factor Rh (D), dosar anticuerpos anti-Rh (D), y administrar la inmunoglobulina Rh (D), cuando corresponda, previene la sensibilización materna y mejora los resultados en neonatos. Los beneficios superan substancialmente los daños.

35- Incompatibilidad $\mathrm{Rh}$, repetir anticuerpos anti-Rh a las 24-28 semanas excepto con padre Rh- (B)

Hay aceptable evidencia de repetir los anticuerpos anti-Rh en mujeres Rh - no sensibilizadas (excepto con padre biológico Rh-) y administrar la inmunoglobulina Rh (D), cuando corresponda. Esto provee beneficios adicionales al primer rastreo en la sensibilización materna y los resultados en neonatos Los beneficios de repetir los anticuerpos anti-Rh superan sustancialmente los daños potenciales. No se encontró nueva evidencia sobre nuevas pruebas de rastreo, nuevos tratamientos o de potenciales daños asociados al rastreo y tratamiento de la incompatibilidad $\mathrm{Rh}$. Sin embargo hay buena evidencia previa de la eficacia y efectividad de tipificar el factor $\mathrm{Rh}$ (D), dosar anticuerpos anti-Rh (D), y administrar la inmunoglobulina $\mathrm{Rh}$ en el posparto.

\section{6- Ambliopía, estrabismo y agudeza visual en niños menores} de 5 años (B)

No se hallo evidencia directa de que el rastreo de problemas visuales mejore la agudeza visual en niños. Sin embargo hay aceptable evidencia de que el rastreo detecta con razonable seguridad ambliopía, estrabismo y vicios de refracción en niños con estos problemas; que el rastreo intensivo comparado al rastreo usual mejora la agudeza visual y que el tratamiento de ambliopía y estrabismo pueden mejorar la agudeza visual y reducir la ambliopía a largo plazo. No se hallaron evidencias de daños potenciales por rastreo, posiblemente pequeños, y se concluyó que los beneficios de rastrear probablemente superen los daños potenciales.

37- Caries en niños preescolares mayores de 6 meses en zonas con insuficiente fluoración del agua por médicos de atención primaria(B)

Hay aceptable evidencia de que en niños preescolares mayores de 6 meses de zonas con insuficiente fluoración del agua la prescripción por médicos de atención primaria, de suplementos fluorados por vía oral reduce la caries dental. Los beneficios de los suplementos fluorados superan los daños potenciales de la fluorosis dental.

38- Caries en preescolares mediante valoración de riesgo por médicos de atención primaria (I)

No se hallaron herramientas o algoritmos validados de valoración de riesgo por médicos de atención primaria y poca evidencia de que los médicos de atención primaria sean capaces de valorar sistemáticamente el riesgo de enfermedad dental en niños preescolares. Hay poca evidencia de que el consejo a padres o la derivación de niños de alto riesgo reduzcan la caries o la enfermedad dental. Hay insuficiente evidencia como para determinar que el balance entre beneficios y daños de la valoración rutinaria de riesgo para prevenir enfermedad dental en preescolares.

\section{9- Escoliosis idiopática en adolescentes (D)}

No se halló evidencia de que el rastreo de adolescentes asintomáticos detecte escoliosis idiopática en un estadio más temprano que sin rastreo. La exactitud de las pruebas de rastreo mas comunes (prueba de flexión hacia delante con o sin escoliómetro) para identificar adolescentes con escoliosis idiopática es variable y el seguimiento de los identificados en programas comunitarios es pobre. Hay aceptable evidencia de que el tratamiento de la escoliosis idiopática en adolescentes produzca beneficios en salud (menor dolor y discapacidad) en solo una pequeña proporción de personas. La mayoría de los casos detectados por rastreo no progresan a formas clínicamente significativas. Es poco probable que las escoliosis detectadas por rastreo requieran tratamientos agresivos como cirugía y hay aceptable evidencia de que pueden producir daños moderados incluyendo el uso innecesario de dispositivos de estabilización de columna y derivaciones inapropiadas a especialistas. Se concluye que los daños del rastreo de escoliosis idiopática en adolescentes exceden los potenciales beneficios.

Agustín Ciapponi [ Unidad de Medicina Familiar y Preventiva, Hospital Italiano de Buenos Aires. Centro Cochrane Argentino, Instituto de Efectividad Clínica y Sanitaria. ]

Referencias

1. Guide to Clinical Preventive Services: Second Edition (1996). Report of the U.S. Preventive Services Task Force

2. Ciapponi A. Nueva guía de cuidados preventivos de la Fuerza de Tareas de Estados Unidos (primera entrega). Evid. actual. práct. Ambul. 2005;8:179-182

3. Ciapponi A, Alderete M, Calvo C. Guía de cuidados preventivos de la USPSTF (3ra edición, 2000-2003). Evid. actual. práct. Ambul. 2003;6:79-84 